\title{
Las revistas colombianas de antropología y ciencias sociales en tiempos de mediciones: desafíos de la comunidad académica
}

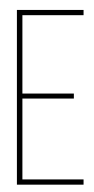

n una nota editorial publicada no hace mucho tiempo, la anterior editora de Antípoda, la profesora Mónica Espinosa (2015), describía la labor editorial en la revista como el resultado de una tensión entre dos polos. El primero, de la pluralidad, marcado por la creciente diversidad interna de la disciplina, y el segundo, de la medición, caracterizado por lo que definió como el "clima actual en el que se desarrollan las actividades de investigación y publicación en las ciencias sociales”, donde se naturalizan ciertas mediciones como una forma de tomar el pulso a la calidad de la publicación científica. Así, por un lado, el editor se enfrenta a un campo de conocimientos cada vez más complejo y diverso, mientras que, por el otro, debe prestar atención a los mecanismos, en su mayoría ajenos, que prometen determinar la calidad de su publicación. Se trata, pues, de publicar en "tiempos de mediciones", con la tarea aún pendiente de reflexionar con mayor vigor sobre la medición como proceso y como mecanismo transformador de la realidad que pretende medir. Basta dar un vistazo somero a la literatura existente sobre la consolidación de estándares y calificaciones para entender que lo que está en juego no es solamente la medición de la calidad de nuestras publicaciones, sino las prácticas editoriales en todos sus niveles. Como bien lo expone la profesora Espinosa, todos estamos por exceso o por defecto "atrapados en una situación que debería ameritar reflexiones más profundas”, además de acciones concertadas entre universidades, áreas de conocimiento y editores.

Desde esa nota editorial algo más ha cambiado en el panorama nacional de la publicación académica. Si bien a partir del 2002 las revistas científicas han sido evaluadas y clasificadas por el Servicio Nacional de Indexación de Publicaciones Especializadas Seriadas de Ciencia, Tecnología e Innovación (Publindex), 
la Convocatoria 768 del $2016^{1}$ de Colciencias modificó los criterios de indexación y homologación de revistas en el marco de la Política Nacional para Mejorar el Impacto de las Publicaciones Científicas Nacionales. Este proceso de evaluación propone un nuevo sistema de clasificación en el que se normaliza - con ciertas correcciones, técnicamente muy discutibles-la medición internacional basada en la citación y afincada en dos grandes indexadores: Thompson Reuters y Elsevier/Scopus ${ }^{2}$. A pesar de las múltiples voces críticas que solicitaron revisiones de la política y la convocatoria, Colciencias no cambió su propuesta en términos importantes. El problema, más allá de una larga discusión técnica sobre la medición en sí misma, es que Colciencias "confunde el objetivo de la política con su indicador”, en palabras de Yuri Jack Gómez. Esto es, imagina que solo con establecer como criterio de clasificación los estándares internacionales las revistas colombianas alcanzarán las metas que el estándar les solicita. De esta manera, más que la medición, lo que está en juego es el modo en la que esta se utiliza para intervenir el campo de las prácticas y las publicaciones científicas en el país.

Las revistas académicas colombianas han sido las primeras en sentir el resultado de estas transformaciones profundas, que en la mayoría de los casos supondrán la pérdida de las categorías o la expulsión del Publindex³. Sin embargo, los efectos de este cambio en la política van mucho más allá y prometen afectar al conjunto de las prácticas académicas nacionales, como los resultados de la medición de grupos, los mecanismos para la condonación de deudas de estudios de posgrado y el escalafonamiento docente, entre otros. En un mundo en el que

1 La Convocatoria 768 de Colciencias presenta como su objetivo evaluar la calidad de las revistas científicas nacionales mediante criterios relacionados con la gestión editorial, la visibilidad y el impacto de las publicaciones, con el propósito de incrementar la calidad de la producción científica nacional y la inserción de esta producción a escala internacional. Como resultado de esta nueva política, centrada en la medición internacional del impacto de las revistas para determinar sus categorías, se estima que más de doscientas revistas científicas nacionales bajarán sus categorías y un número no despreciable de publicaciones colegas directamente quedarán expulsadas de Publindex. Para conocer los términos de la Convocatoria 768, véase http://www.colciencias.gov.co/convocatorias/investigacion/convocatoria-para-indexacion-re vistas-cientificas-colombianas.

2 Thomson Reuters y Elsevier/Scopus son las bases de datos privadas más comúnmente empleadas en la academia para conocer y medir los factores de impacto bibliográfico. La primera es una firma multinacional de medios e información y la segunda es una empresa global de análisis de información. Sobre el multimillonario negocio de las publicaciones seriadas científicas, véase el reciente artículo en The Guardian, "Is the Staggeringly Profitable Business of Scientific Publishing Bad for Science?”, 27 de junio del 2017. https://www.theguardian.com/ science/2017/jun/27/profitable-business-scientific-publishing-bad-for-science.

3 Los resultados definitivos de la convocatoria fueron publicados en la Resolución 0929 del 15 de septiembre del 2017. El total de revistas participantes fue 627, de las cuales se avalaron 538, pero solo 244 fueron clasificadas. Por categoría, solo una revista quedó clasificada en A1, 14 en la categoría A2, 104 en B y 125 en C. http://www.colciencias.gov.co/convocatorias/ investigacion/convocatoria-para-indexacion-revistas-cientificas-colombianas. 
se ha vuelto cada vez más tangible el dicho "publica o perece”, la existencia, calidad, permanencia y vitalidad de las publicaciones científicas deberían ser un motivo de preocupación para todos los científicos colombianos.

En este contexto de incertidumbres, las revistas de antropología del país iniciamos una serie de acercamientos con el fin de reflexionar en conjunto sobre el panorama editorial en el que vivimos y buscar mecanismos de colaboración y fortalecimiento. Colciencias y Publindex fueron un detonante para reunirnos y tomarle el pulso al contexto heterogéneo de preocupaciones que compartimos como editores. Una primera conclusión importante es que los factores problemáticos que enfrentamos exceden con creces la coyuntura actual alrededor de Publindex, por lo que esperamos que este espacio se convierta en un escenario en el que podamos compartir, mejorar y ampliar nuestras buenas prácticas editoriales. Se trata de ofrecer a la academia nacional e internacional revistas de alta calidad que les permitan a los autores y lectores entablar el tipo de diálogos que enriquezcan, complejicen nuestras disciplinas y nos den la posibilidad de desarrollar acciones articuladas con otros sectores sociales.

A continuación, enumeraremos algunos de los factores problemáticos que constituyen nuestras preocupaciones cotidianas como editores, organizadas en tres bloques:

\section{Colciencias-Publindex y la medición}

Los editores de las revistas científicas colombianas hemos insistido en el clima de incertidumbre actual alrededor de Publindex. Por una parte, debido a los cambios permanentes en la gestión misma del aplicativo: Publindex les impone a los equipos editoriales cargas significativas de trabajo, que se duplican cuando el aplicativo falla, y al mismo tiempo no es del todo claro qué beneficio representa para las publicaciones estar en este sistema de indexación. Por otra parte, nos preocupan los efectos no necesariamente planeados que la actual medición puede acarrear, pues en algunos casos lo que está en juego es la existencia misma de nuestras publicaciones, toda vez que a causa de la pérdida de las categorías existen dudas sobre la continuidad de la financiación de muchas revistas.

Los editores concordamos en las bondades de la medición de calidad para las publicaciones científicas, en la medida en que es una herramienta de evaluación del propio trabajo. Sin embargo, es preciso señalar que la medición de calidad 
no equivale a la medición citacional ${ }^{4}$. En este sentido, mantenemos importantes diferencias técnicas con respecto a cómo se efectúa la reciente asignación de categorías a las revistas, basada en los índices citacionales internacionales Journal Citation Report (JCR), Scimago Journal Rank (SJR) e índice H (para las categorías inferiores). Al respecto, consideramos que, dado que los indicadores de impacto son por definición fluctuantes y no necesariamente mantienen vinculación con la calidad y necesidad de las investigaciones que se producen y publican en el país, no pueden ser el eje determinante en la asignación de las categorías que establece Publindex. Entonces, cuestionamos la idoneidad de la nueva política de indexación nacional, en la medida en que no considera acciones efectivas de fomento a la calidad, pues se restringe a la homologación de las mediciones y calificaciones internacionales de las revistas. Por ello, en repetidas ocasiones hemos solicitado a Colciencias la ampliación del reconocimiento a otros índices y parámetros, con miras a incluirlos en los criterios de evaluación. Un ejemplo de esto son los resultados y propuestas surgidas de los encuentros colombianos y latinoamericanos de revistas en ciencias sociales y humanidades realizados en el 2015 y el $2016^{5}$.

Las publicaciones hacen parte del contexto académico nacional, y las relaciones entre ambos son amplias y diversas. Como editores, podemos entrever las consecuencias más allá de las publicaciones en este escenario. Por ello vale la pena preguntarse si los cambios en Publindex están articulados con transformaciones más generales en la financiación y el control de los académicos colombianos.

Por otra parte, las políticas del Publindex están basadas exclusivamente en la medición del impacto de las publicaciones colombianas; solicitan información excesiva y por fuera de los objetivos y del quehacer de una revista científica y buscan el logro de resultados en los que las calificaciones parecen tener mayor relevancia que lo académico. Publindex demanda de las revistas el ajuste a estándares internacionales, con elaboración de metadatos exportables para los

4 La producción y divulgación de conocimiento tanto en las ciencias sociales como en otras áreas no se reduce a la generación de citas, sino que también tiene una función y proyección social. Muchos de nuestros lectores se desempeñan por fuera del ámbito académico y emplean las revistas como referencia, aunque ello no genere citación.

5 Véanse http://www.icesi.edu.co/unicesi/2015/05/29/primer-encuentro-colombiano-de-revistas -de-ciencias-sociales-y-humanidades/, http://www.icesi.edu.co/unicesi/2015/11/09/primer-en cuentro-latinoamericano-y-del-caribe-de-revistas-de-ciencias-sociales/ y http://www.icesi. edu.co/unicesi/2016/09/02/i-foro-regional-sobre-revistas-cientificas-politicas-visibilidad-yacceso-abierto/. 
índices y bases bibliográficas, pero utiliza en su sistema un formulario manual de adquisición de datos, con menús incompletos, sin ninguna posibilidad de exportación de metadatos, con dificultades importantes en el almacenamiento y sin un sistema de consultas o reportes bibliográficos.

Lo anterior nos permite concluir que Publindex no es un índice para el investigador, ni una base de consulta con estándares internacionales, y no aparece como una opción cuando se busca una revista, un autor o un artículo en buscadores genéricos. Sistemas eficientes y autoactualizables, como Orcid, son, a su vez, compatibles con bases de datos como SciELO y Redalyc, lo que permite que se generen informes sobre la producción de autores, instituciones y revistas. Por lo anterior, cabe preguntarse acerca de la utilidad de Publindex y por qué Colciencias no abandona su actual e ineficaz sistema e implementa un modelo eficiente, e incluso con mayor alcance "internacional” y regional.

En este orden de ideas, son absolutamente relevantes la formación de editores académicos y la implementación de estrategias para promover la selección y revisión por pares. Estas acciones y otras iniciativas deberían ser promovidas directamente por Colciencias. Sin embargo, aunque han sido vagamente mencionadas por la institución, están lejos de contar con un cronograma y coordinación en su agenda.

Actualmente, son pocas las revistas en Colombia que cuentan con protocolos XML, necesarios para posicionar la información de los artículos en los buscadores científicos y entrar en bases de datos de amplio espectro. De la misma manera, continuamos siendo poco competitivos con respecto a revistas de otras geografías, debido en parte a que los tiempos editoriales siguen siendo más lentos, con acceso restringido a contenidos y evaluadores internacionales. Las políticas de acceso abierto, que han mostrado ampliamente sus bondades, todavía llevan a un número importante de revistas latinoamericanas y nacionales a competir en condiciones desiguales en relación con aquellas muy posicionadas (publicadas, casi siempre, en lengua inglesa), que cuentan con recursos económicos y cobran a sus usuarios por el uso de los contenidos.

Por otra parte, los índices bibliométricos, como medida para evaluar la calidad y el impacto de la ciencia y de los investigadores, continúan subestimando trabajos que pueden tener un alto impacto a largo plazo. En este sentido, debemos adoptar prácticas que, además de considerar las calificaciones cuantitativas, afinen la inclusión de indicadores cualitativos que les permitan a los equipos editoriales identificar calidad e innovación. 


\section{Apoyo institucional}

La labor del editor en el país es escasamente reconocida. Muy pocas publicaciones tienen un editor de tiempo completo, y muchas instituciones no cuentan con los recursos más básicos para llevar a cabo buenas prácticas editoriales.

En este contexto, en la figura del editor se multiplican las tareas, muchas de ellas más allá de la labor editorial propiamente dicha. Son muy pocas las revistas que funcionan con equipos editoriales y, en varias, el relevo continuo de personal es común, lo cual incide en la calidad de la publicación.

El apoyo entre revistas es muy escaso y existe una idea de la competencia en el gremio que impide prácticas de comunicación efectiva entre editores. Por ello es indispensable mejorar el diálogo entre revistas colegas e imaginar modos de colaboración para el fortalecimiento mutuo.

\section{Apoyo gremial}

En nuestra función como órganos de divulgación del conocimiento y la investigación, las revistas debemos redoblar nuestros esfuerzos con miras a generar espacios de debate y lectura que excedan la publicación de artículos. La vitalidad de nuestra academia se puede medir por nuestras revistas, pero no solo en la calidad de los artículos originales, sino también en la importancia relativa de secciones como reseñas, cartas al editor y comunicaciones cortas.

No obstante, la coyuntura de la Convocatoria 768 pone de manifiesto lo que los editores identificamos como una ausencia de apoyo por parte del gremio antropológico nacional. En general, los editores encontramos más colaboración en autores y evaluadores extranjeros que en los colegas nacionales.

Las revistas necesitan de los académicos y de la academia para subsistir. A largo plazo es importante modificar la manera en la que los antropólogos colombianos nos leemos y nos comunicamos a través de nuestras publicaciones.

Con este comunicado, las revistas expresamos nuestra profunda preocupación por el actual modelo de indexación implementado por Publindex mediante la Convocatoria 768, y hacemos un llamado a los colegas antropólogos y de las ciencias sociales a sumarse al esfuerzo conjunto de fortalecer nuestro quehacer académico y pedagógico, y a profundizar nuestra responsabilidad social y política frente al avance de las políticas actuales de ciencia y tecnología que, en vez 
de fomentar y fortalecer la labor científica del país, lesionan los proyectos editoriales y académicos.

Bogotá, 18 de agosto del 2017

\section{Firman:}

Antípoda (Universidad de los Andes)

Revista Colombiana de Antropología (Instituto Colombiano de Antropología e Historia)

Tabula Rasa (Universidad Colegio Mayor de Cundinamarca)

Universitas Humanística (Pontificia Universidad Javeriana)

Maguaré (Universidad Nacional de Colombia)

Jangwa Pana (Universidad del Magdalena)

Boletín de Antropología (Universidad de Antioquia)

Virajes (Universidad de Caldas)

Mundo Amazónico (Instituto Amazónico de Investigaciones [Imani], Universidad Nacional de Colombia; Programa de Pós-Graduação em Antropologia Social, Universidade Federal do Amazonas)

Fronteras de la Historia (Instituto Colombiano de Antropología e Historia)

Anuario Colombiano de Historia Social y de la Cultura (Departamento de Historia, Universidad Nacional de Colombia)

Revista Colombiana de Sociología (Departamento de Sociología, Universidad Nacional de Colombia)

Nómadas (Fundación Universidad Central)

Revista de Estudios Sociales (Universidad de los Andes)

Revista CS (Facultad de Derecho y Ciencias Sociales de la Universidad Icesi)

Revista Ciencias y Humanidades (Centro de Estudios en Ciencias y Humanidades, Medellín)

Misión Jurídica (Universidad Colegio Mayor de Cundinamarca) 